Please cite this article in press as: Pellicciari MC, et al., Increased cortical plasticity in the elderly: Changes in the somatosensory cortex after paired associative stimulation, Neuroscience (2009), doi: 10.1016/j.neuroscience.2009.06.013

Neuroscience $\mathrm{xx}(2009) \mathrm{xxx}$

\title{
INCREASED CORTICAL PLASTICITY IN THE ELDERLY: CHANGES IN THE SOMATOSENSORY CORTEX AFTER PAIRED ASSOCIATIVE STIMULATION
}

\author{
M. C. PELLICCIARI, ${ }^{a}$ C. MINIUSSI, ${ }^{\mathrm{a}, \mathrm{b}}$ P. M. ROSSINI ${ }^{\mathrm{c}, \mathrm{d}, \mathrm{e}}$ \\ AND L. DE GENNARO ${ }^{*} *$ \\ AQ: $1{ }^{a}$ Cognitive Neuroscience Section, IRCCS San Giovanni di Dio Fate- \\ benefratelli, Brescia, 25125, Italy \\ ${ }^{\circ}$ Department of Biomedical Sciences and Biotechnologies, National \\ Institute of Neuroscience, University of Brescia, Brescia, Italy \\ ${ }^{c}$ Neurology Clinic, University Campus Biomedico, Rome, Italy \\ ${ }^{d}$ Department of Neuroscience, AFaR Isola Tiberina Fatebenefratelli, \\ Rome, Italy \\ ${ }^{\circ}$ Casa di Cura e IRCCS S. Raffaele Cassino, Rome, Italy \\ ${ }^{f}$ Department of Psychology, University of Rome "Sapienza," Rome, Italy
}

\begin{abstract}
A fundamental feature of the human cortex is the capability to express plastic changes that seem to be present even during physiological aging. The paired associative stimulation (PAS) protocol is a paradigm capable of inducing neuroplastic changes, possibly by mechanisms related to spike timing-dependent associative neuronal activity, and represents a suitable tool for investigating age-dependent neuroplastic modulations of the primary somatosensory cortex (S1). To examine age dependency of S1 plasticity, the amplitude changes of median nerve somatosensory evoked potential (SEP) before and after PAS intervention were investigated in young and elderly subjects. The main finding of our study is that low-frequency medial nerve stimulation paired with transcranial magnetic stimulation over the contralateral cortex enhances S1 excitability. Moreover, the S1 long term potentiation-like plasticity changes as a function of aging, with a significant increase of N20-P25 complex in the elderly compared to young subjects. These results are congruent with the hypothesis that some elderly subjects retain a high level of plasticity in specific neuronal circuits. Such plasticity could represent a compensatory mechanism, in terms of functional reserve of somatosensory cortex, used by the aging brain to counterbalance the cortical degeneration associated with aging. () 2009 Published by Elsevier Ltd on behalf of IBRO.
\end{abstract}

Key words: age, somatosensory cortical plasticity, paired associative stimulation, TMS.

Several studies have suggested that the cerebral cortex is not a static structure but has dynamic organization asso-

*Corresponding author. Tel: +39-06-49917647; fax: +39-06-4451667. E-mail address: luigi.degennaro@uniroma1.it (L. De Gennaro).

Abbreviations: ANCOVA, analysis of covariance; ANOVA, analysis of variance; APB, abductor pollicis brevis; EEG, electroencephalogram or -graphy or -graphic; EMG, electromyogram or -graphy or -graphic; EOG, electrooculogram or -graphy or -graphic; LTP, long-term potentiation; MEP, motor evoked potential; MN, median nerve; M1, primary motor cortex; PAS, paired associative stimulation; RMT, resting motor threshold; SEP, somatosensory evoked potential; S1, primary somatosensory cortex; TMS, transcranial magnetic stimulation. ciated with mechanisms of plasticity (Recanzone et al., 1992; Rossini et al., 1994a; Castro-Alamancos et al., 1995; Sanes and Donoghue, 2000; Stavrinou et al., 2007; Mercado, 2008). One mechanism that could be responsible for cortical plasticity is the modulation of the synaptic strength of cortical horizontal connections by long-term potentiation (LTP) and long-term depression (LTD) (Castro-Alamancos et al., 1995; Rioult-Pedotti et al., 1998; Sanes and Donoghue, 2000).

Cortical plasticity appears to be reduced during physiological aging in association with extensive neurochemical and neurophysiologic changes (Burke and Barnes, 2006; Rossini et al., 2007). Normal aging is associated with a loss of synaptic contacts and a relative decrease in the excitability of intracortical inhibitory circuits (Peinemann et al., 2001; Hortobagyi et al., 2006; Oliviero et al., 2006), which then cause a decline in sensory processing, motor performance and cognitive function. Moreover, age-dependent synaptic plasticity is highlighted by behavioral impairments correlated with region-specific changes in dendritic morphology, cellular connectivity, and gene expression (Godde et al., 2002; Sawaki et al., 2003; Burke and Barnes, 2006; Mora et al., 2007; Ward et al., 2007).

Plastic cortical changes can be investigated with paired associative stimulation (PAS), which is shaped like associative LTP in animal studies (Markram et al., 1997; Stefan et al., 2002; Classen et al., 2004). This protocol couples a single electric stimulus delivered at specific time intervals to a peripheral nerve with a single transcranial magnetic stimulation (TMS) pulse over primary motor cortex (M1) or primary somatosensory cortex (S1), causing changes in their excitability (Mariorenzi et al., 1991; Stefan et al., 2002; Wolters et al., 2003). Therefore it is believed that this repetitive associated stimulation can induce LTPlike phenomena.

Specifically, LTP occurs if the action potential follows an excitatory post-synaptic potential elicited by sub-threshold afferent stimulation within a few milliseconds (Markram et al., 1997; Bi and Poo, 1998; Feldman, 2000). PASinduced plasticity evolves rapidly and is persistent, topographically specific, and reversible (Stefan et al., 2002). An involvement of LTP-like processes is supported by all these properties and by its blockade by NMDA receptor AQ: 2 antagonists (Malenka and Nicoll, 1993). These LTP-like changes can be quantified by recording motor evoked potential (MEP) (Stefan et al., 2002; Wolters et al., 2003; Ziemann, 2004; Fratello et al., 2006) or somatosensory evoked potential (SEP) (Wolters et al., 2005; Litvak et al., 2007) amplitude. The site of PAS-action has been local-

doi:10.1016/j.neuroscience.2009.06.013 
ized to the cortex (Stefan et al., 2002; Wolters et al., 2003) but mechanisms at the level of the spinal cord may contribute to PAS-induced changes in corticomotor excitability (Meunier et al., 2007).

The PAS protocol has been used to investigate the effects of physiological aging on motor cortex plasticity (Muller-Dahlhaus et al., 2008; Tecchio et al., 2008). However, age-dependent changes in cortical plasticity have never been investigated in S1.

The main aim of present study was to investigate the functional modulation of S1 cortical plasticity during physiological aging. Hence, we investigated differences between young and elderly healthy subjects by evaluating LTP-like changes related to spike-timing dependent plasticity of neuronal synapses and induced by the PAS protocol on SEP component amplitude.

The influence of gender was also taken into account, since (Tecchio et al. 2008) showed that PAS-induced motor cortex plasticity is gender-dependent.

\section{EXPERIMENTAL PROCEDURES}

\section{Subjects}

Thirty-two healthy volunteers subjects took part in the experiment. They were divided into two groups: 16 elderly subjects [eight males and eight females; mean age and standard error ( \pm SE) $62.1 \pm 1.5$ years] and 16 young subjects (eight males and eight females; mean age $26.2 \pm 0.8$ years). Elderly subjects were selected from an institute's normative archive, whereas young subjects were selected from a university student population. All subjects reported themselves as right-handed and had no history of peripheral or neurological impairment. In particular, exclusion criteria were: cognitive decline or memory disturbance ever experienced, previous transient ischemic attack or stroke, brain injury, alcohol abuse or drug addiction and contraindications to TMS (Wassermann, 1998). All subjects were informed about the experimental procedures and provided written informed consent. The experimental protocol was approved by the Ethics Committee of IRCCS Centro San Giovanni di Dio Fatebenefratelli, Brescia, Italy.

\section{Study design}

The principal aim of the experiment was to determine the effect induced by PAS protocol on SEPs recorded from the scalp positions overlying $S 1$ in elderly and young subjects. Participants were seated in a comfortable chair in a Faraday cage soundproof room. They were required to keep their right arm completely relaxed and to pay attention to the stimulated hand during the entire experimental session (Stefan et al., 2004). All subjects were tested within the same time period of the day (10:30 AM to 1:30 PM), with the aim to control for potential circadian effects (Sale et al., 2007).

\section{TMS}

TMS was applied using a figure-of-eight double $70 \mathrm{~mm}$ coil connected to a Magstim SuperRapid magnetic stimulator (Magstim, Whitland, UK). The coil was placed tangentially to the scalp with the handle pointing backwards and laterally at about a $45^{\circ}$ angle away from the midsagittal axis of the subject's head. The optimal site of stimulation for eliciting MEPs in the right abductor pollicis brevis (APB), termed the "motor hotspot," was chosen by positioning the coil approximately over the central sulcus and moving it on the scalp in $0.5 \mathrm{~cm}$ steps over M1 of the left cortex, assessed at a moderately suprathreshold stimulation intensity and marked directly on the scalp with a soft-tip pen. On this site, the resting motor threshold (RMT) was determined as the stimulator intensity needed to produce a response of at least $50 \mu \mathrm{V}$ in amplitude in the relaxed APB in at least five of 10 consecutive stimulations at a resolution of $1 \%$ of the maximal stimulator output (Rossini et al., 1994b). Complete muscle relaxation was monitored throughout the experiment and regulated by audiovisual feedback.

\section{Electrical somatosensory stimulation}

Electrical nerve stimulation used to induce SEPs was performed with a constant current electrical stimulator (STM140 rev 1.0, HTL, Udine, Italy) with standard parameters (cathode proximal). The AQ: 3 stimulating electrode was placed on the right median nerve (MN) at the level of the wrist. MN stimulation was performed using a pulse with a $200 \mu \mathrm{s}$ width at a frequency of $3 \mathrm{~Hz}$ and a stimulation intensity of $\sim 300 \%$ of the individual perceptual threshold (Wolters et al., 2005). The hand representation at S1 ("somatosensory hot-spot") was marked $2 \mathrm{~cm}$ posterior to $\mathrm{C} 3$ position, corresponding to CP3 (Nuwer et al., 1994; Wolters et al., 2005; Litvak et al., 2007). Before and after PAS intervention, 500 electric stimuli were recorded (500 pre-PAS +500 post-PAS). During PAS intervention, the same parameters of nerve stimulation were utilized.

\section{PAS}

The PAS protocol reproduces the experimental procedure of Wolters et al. (2005), which represented a modification of PAS protocol generally used for the M1 area (Stefan et al., 2002). The protocol consisted of single electrical stimuli delivered to the right $\mathrm{MN}$ at the level of the wrist at $300 \%$ of the perceptual threshold and followed by TMS delivered over the hand representation of $\mathrm{S} 1$. Namely, the left $\mathrm{S} 1$ was stimulated by placing the central area of the junction of the two coil wings at a scalp site on CP3 along a line parallel to the midline with induced current directed in a posterior-anterior axis. TMS was applied at an intensity of 1.3 times the RMT. The interval between MN stimulation and the subsequent TMS pulse was $20 \mathrm{~ms}$. This interval is the modal time for an afferent signal arising from MN at the level of the wrist to travel to S1 (i.e. N20 latency) (Wolters et al., 2005). One hundred forty pairs of stimuli were delivered at $0.1 \mathrm{~Hz}$ over $25 \mathrm{~min}$. During PAS, subjects were asked to watch their own right hand, since this condition has been demonstrated to give the maximal PAS-induced plasticity (Classen et al., 2004). The entire experimental time session lasted $30 \mathrm{~min}$.

\section{Recording}

Electroencephalographic (EEG) signals were recorded from 12 sites using TMS-compatible $\mathrm{Ag} / \mathrm{AgCl}$-coated electrodes mounted on an elastic cap (EasyCap GmbH, Herrsching, Germany). TMScompatible EEG equipment (BrainAmp DC/MRplus, Brain Products $\mathrm{GmbH}$, Munich, Germany) was used to record continuous EEG activity during PAS. The montage included four midline sites $(\mathrm{Fz}, \mathrm{Cz}, \mathrm{Pz}, \mathrm{Oz})$ and eight sites over each hemisphere (C4, C3, $\mathrm{CP} 1, \mathrm{CP} 2, \mathrm{CP} 3, \mathrm{CP} 4, \mathrm{CP} 5, \mathrm{CP} 6)$ and left and right mastoid (M1, M2) positioned according to the International $10-20$ system. Additional electrodes were used as the ground and reference. The ground electrode was placed in the midfrontal (Fpz) position. The reference electrode was placed at the tip of the nose during EEG recording. Recordings obtained from an M1 and M2 electrode were used off-line to re-reference the scalp recordings to the conventional linked mastoids (i.e. average of M1 and M2).

Horizontal and vertical eye movements were detected by recording electrooculograms (EOGs) in order to monitor subject behavior on-line and reject, off-line, trials with ocular artefacts. Surface electromyography (EMG) activity was recorded from the right APB muscle with the active electrode mounted on the belly muscle and the reference electrode placed over the base of the metacarpophalangeal joint of the thumb. EEG, EOG and EMG 
signals were acquired with a band-pass filter at $0.02-1000 \mathrm{~Hz}$, and then filtered off-line at $0.2-1000 \mathrm{~Hz}$ for EEG, EMG and $0.02-15 \mathrm{~Hz}$ for EOG. All signals were digitized at a sampling rate of $5 \mathrm{kHz}$ using a $16 \mathrm{~b}$ A/D-converter. The skin-electrode impedance was kept below $5 \mathrm{k} \Omega$.

\section{Data and statistical analysis}

The optimal site for SEP analysis was the CP3 electrode, which was the electrode with the largest amplitude response, likely dominated by $\mathrm{S} 1$ activity. The epoching of the MN SEPs was performed off-line. All data collected were epoched between -50 and +100 ms relative to the MN stimulation. Epochs where voltage exceeded $\pm 100 \mu \mathrm{V}$ at the VEOG and HEOG electrodes or those containing artefacts were rejected. All amplitude values were calculated with reference to the $50 \mathrm{~ms}$ pre-stimulus baseline. Measurement windows were determined from an inspection of the group grand-average waveform. The P14 amplitude was measured as the difference between the baseline and the first positive peak occurring at a latency of around 13-18 ms after the time of MN stimulation. The amplitude of N20 was measured as the first negative peak between 18 and 23 ms. The N20-P25 complex was determined as the difference between the N20 peak and the subsequent positivity peak (P25), occurring at a latency of around 23-29 ms. P25 amplitude was subsequently calculated as the difference between the amplitude of the N20-P25 complex and the N20 amplitude. Finally, the N30 and P40 peak amplitudes were measured between 29 and $38 \mathrm{~ms}$ and between 38 and 45 ms, respectively.

According to previous studies (Wolters et al., 2005; Murakami et al., 2008; Litvak et al., 2007; Tamura et al., 2009), the statistical analyses compared absolute amplitude values recorded beforePAS to those after-PAS intervention. The main dependent variable was the amplitude of the N20-P25 complex. A three-way mixed design analysis of variance (ANOVA), age group (young vs. elderly) $\times$ gender (male vs. female) $\times$ condition (pre- vs. post-PAS) was carried out.

The same ANOVA was also performed on the amplitude of the P14, N30, and P40 components. To assess significant interactions, selected two-sample comparisons were performed by means of one-tailed $t$-tests. The one-tailed probability has been used since an a priori directional hypothesis does exist with respect to the increase of MEP amplitude. The appropriate $t$-tests have been used according to the repeated/non-repeated factors. The Bonferroni correction was applied to correct for multiple comparisons. Considering the mean correlation between the dependent variables $(r=0.70)$, the alpha level was then adjusted to $\leq 0.03$ (Sankoh et al., 1997).

\section{RESULTS}

The PAS intervention affected SEPs in the time-window between 20 and $25 \mathrm{~ms}$ in both groups, inducing an average increase of the N20-P25 complex by $5.4 \%$ in young and by $9.4 \%$ in elderly subjects (Fig. 1 ).

The main effects and interactions of the ANOVAs on the main dependent variables are reported in Table 1. The T1 N20-P25 complex was significantly affected by aging, with the elderly population having a larger amplitude (5.87 $\mathrm{SE}= \pm 0.74 \mu \mathrm{V})$ than the young $(4.20 \mathrm{SE}= \pm 0.33 \mu \mathrm{V})$ subjects. A robust effect of increased amplitude was found after the PAS intervention (pre-PAS $=4.86 \mathrm{SE}= \pm 0.41 \mu \mathrm{V}$; post-PAS $=5.20 \mathrm{SE}= \pm 0.44 \mu \mathrm{V}$ ). As shown in Fig. 2, both $\mathrm{F} 2$ aging and gender influence this increase. Indeed, the significant Age group $\times$ Condition interaction showed that the post-PAS increase of the N20-P25 amplitude was larger in the elderly (one-tailed paired $t$-test: $t_{15}=5.23 ; P<0.0001$ )

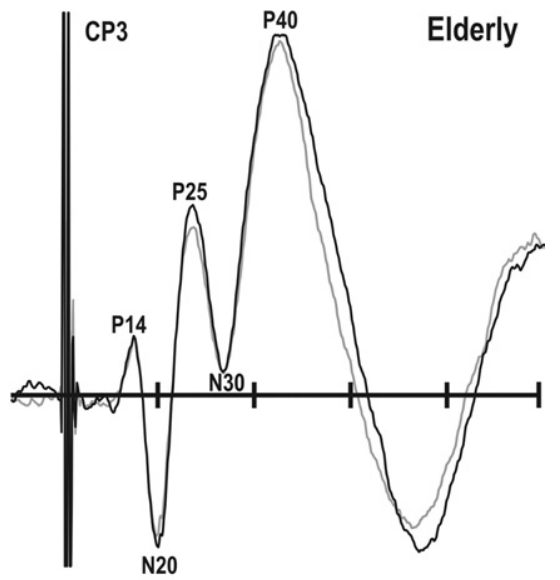

Young
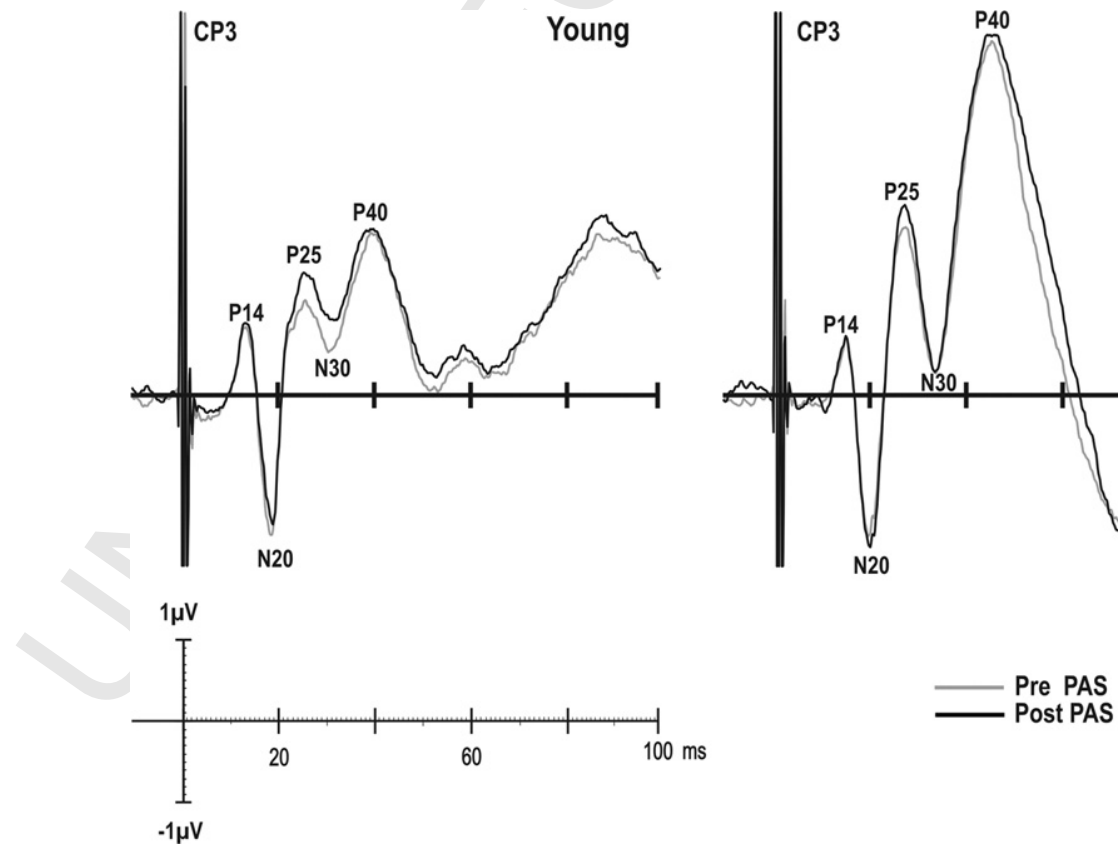

Post PAS

Fig. 1. Grand average waveforms of SEPs following right MN stimulation before and after intervention with PAS of 16 young (left) and 16 elderly (right) subjects. Two traces are superimposed: the thin line represents the response before PAS, the thick line shows the response after PAS. Amplitude of N20/P25 component increases after PAS, whereas others components were unaffected by intervention. Notably, the amplitude of all components of SEP is greater in the elderly than in young subjects. It should be noted that there is a discrepancy between the samples of grand average ERP waveforms figure and real data. This is due to the fact that for data analysis amplitude of components is measured at single subject peak latency (see Experimental Procedures), while for the figure this factor cannot be considered and therefore the time jitter of those peaks results in a smoothed figure. 
Table 1. Results of the Age group $\times$ Gender $\times$ Condition ANOVAs on amplitudes of N20-P25 complex, and of N20 and P25 components

\begin{tabular}{|c|c|c|c|c|c|c|}
\hline & \multicolumn{3}{|l|}{$F_{1,28}$} & \multicolumn{3}{|l|}{$P$} \\
\hline & N20-P25 & N20 & P25 & N20-P25 & N20 & P25 \\
\hline Age group $(A)$ & 4.58 & 0.50 & 5.49 & 0.04 & 0.49 & 0.03 \\
\hline Gender (G) & 3.91 & 2.42 & 2.64 & 0.06 & 0.13 & 0.11 \\
\hline Condition (C) & 38.66 & 1.83 & 8.86 & $<0.001$ & 0.19 & 0.006 \\
\hline$A \times G$ & 0.39 & 2.23 & 0.008 & 0.54 & 0.15 & 0.93 \\
\hline$A \times C$ & 6.77 & 5.46 & 0.33 & 0.01 & 0.03 & 0.57 \\
\hline $\mathrm{G} \times \mathrm{C}$ & 5.59 & 1.28 & 0.25 & 0.02 & 0.27 & 0.62 \\
\hline$A \times G \times C$ & 3.57 & 0.37 & 0.49 & 0.07 & 0.55 & 0.49 \\
\hline
\end{tabular}

than in young subjects $\left(t_{15}=2.49 ; P=0.01\right)$. The significant Gender $\times$ Condition interaction indicated that PAS induced an increase of the N20-P25 complex in females, with a larger effect size $\left(t_{15}=4.83 ; P<0.0001\right.$, Cohen's $\left.d=1.24\right)$ than in male subjects $\left(t_{15}=2.76 ; P=0.007\right.$, Cohen's $\mathrm{d}=0.79$ ). Notably, the triple interaction, although not significant $(P<0.10)$, suggested that the increase of the N20P25 amplitude was significant in young females $\left(t_{7}=1.85\right.$; $P=0.05$ ) and in both elderly groups (males and females $t_{7}=2.24 ; P=0.03$ and $t_{7}=7.73 ; P<0.0001$, respectively), but not in young males $\left(t_{7}=1.56 ; P=0.08\right)$.

The extent of the individual PAS-induced effects on N20-P25 amplitude, expressed as a percentage of pre-

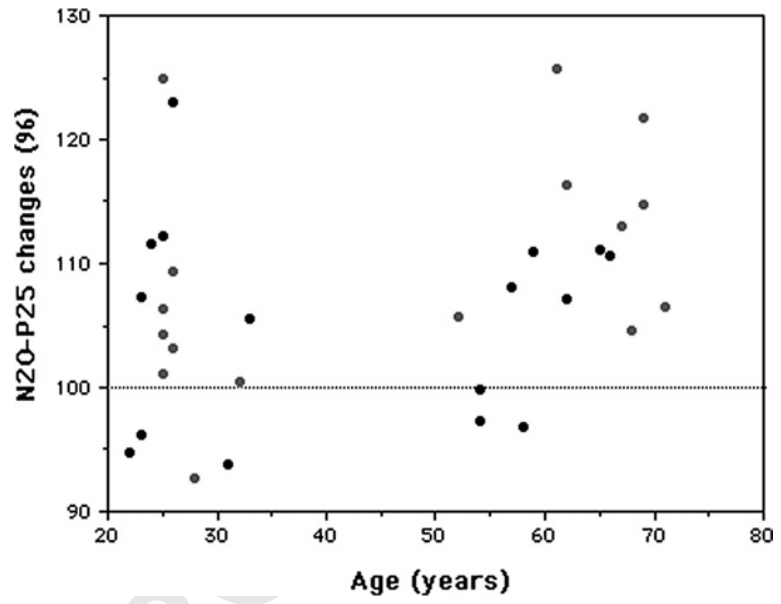

Fig. 3. Scattergram of individual PAS-induced effects on N20-P25 amplitudes expressed as a percentage of pre-PAS condition (postPAS/pre-PAS) as a function of age. Zero corresponds to the pre-PAS N20-P25 amplitude. Grey circles indicate female subjects and black circles represent the male subjects.

PAS condition (post-PAS/pre-PAS), is detailed in Fig. 3. F3 The scattergram shows the between-group differences and confirms the large inter-subject variability. Furthermore, it suggests the existence of a linear relationship between aging and PAS changes, a property limited to

Fig. 2. Effect of PAS on amplitude (expressed in $\mu \mathrm{V}$ ) of the N20-P25 complex, N20, and P25 component of SEP, in 16 male and 16 female subjects
(eight young and eight elderly in each group). The bars illustrate the mean amplitude in pre-PAS (grey bars) and post-PAS (black bars); error bars represent the standard errors. 
elderly subjects. The product-moment correlation between age and N20-P25 changes was $r=0.48(P=0.06)$ in elderly subjects and $r=0.18(P=0.51)$ in young subjects.

Since the average resting motor (young $=52.5 \% \pm 1.8 \%$ and elderly $\left.=58.3 \% \pm 2.2 \% ; F_{1,30}=4.12 ; P=0.05\right)$ and perceptual thresholds (young $=2.5 \pm 0.2 \mathrm{~mA}$ and elderly $=3.1 \pm$ $0.1 \mathrm{~mA} ; F_{1,30}=6.94 ; P=0.01$ ) recorded with magnetic and electrical stimulations were higher in the elderly than in young subjects, these differences may have influenced the magnitude of PAS-induced changes. Hence, we have verified the possible effects of both thresholds values on N20-P25 changes after PAS, and no correlation was significant (motor threshold: $r=0.13, P=0.48$; perceptual threshold: $r=0.21, P=0.24)$. Moreover, we have performed an analysis of covariance (ANCOVA) on the N20-P25 amplitude taking the motor and perceptual thresholds as covariates. The ANCOVA confirmed the effects for the Age group $\left(F_{1,26}=9.57, P=0.005\right)$ and Condition factors $\left(F_{1,26}=\right.$ $35.90, P<0.001)$, the Age group $\times$ Condition $\left(F_{1,26}=6.29\right.$, $P=0.02)$, and Gender $\times$ Condition $\left(F_{1,26}=5.20, P=0.03\right)$ interactions, pointing to a lack of thresholds influence on PAS effects.

Since baseline SSEPs were higher in elderly than in young subjects $\left(F_{1,28}=3.92 ; P=0.05\right)$, these differences may have influenced the magnitude of PAS-induced changes. Nevertheless, the relative increase in the N20P25 amplitude after PAS does not correlate with the N20P25 amplitude at pre-interventional baseline $(r=-0.10$; $P=0.60$ ). As a further control for specificity of PAS effects, we have performed an ANCOVA on percentages of the N20-P25 amplitude changes, considering the pre-PAS N20-P25 amplitude as a covariate. The ANCOVA substantially confirmed the effects for the Age group $\left(F_{1,27}=\right.$ $3.78, P=0.06)$ and Gender factors $\left(F_{1,27}=4.69, P=0.04\right)$. The effect of the covariate also was close to the significance $(\beta=-0.34 ; t=0.07)$.

As a final control for the specificity of PAS effects, we verified the influence of possible differences in conduction slowing in afferent pathways in elderly subjects since the timing between the peripheral and central stimuli in the PAS paradigm critically influences the direction and magnitude of induced changes (Hebb, 1949; Cooke and Bliss, 2006).

These differences do appear to be affected by aging and gender. The two-way ANOVA of Age group (young vs. elderly) $\times$ Gender (male vs. female) on the N20 latency before the PAS protocol pointed to a significant effect for the Age group (young $=19.05 \pm 0.24 \mathrm{~ms}$, elderly $=20.24 \pm 0.29$ $\mathrm{ms} ; F_{1,28}=17.87, P=0.0002$ ) and for Gender (males $=20.32 \pm$ $0.23 \mathrm{~ms}$, females $=18.96 \pm 0.27 \mathrm{~ms} ; F_{1,28}=23.53, P<0.001$ ).

As a consequence of these differences, we have carried out an ANCOVA on the N20-P25 amplitude, considering the $\mathrm{N} 20$ latency as a covariate. The ANCOVA confirmed the effects of the Age group $\left(F_{1,27}=7.18, P=0.01\right)$ and Condition factors $\left(F_{1,27}=37.28, P<0.001\right)$, for the Age group $\times$ Condition $\left(F_{1,27}=6.53, P=0.02\right)$, and Gender $\times$ Condition $\left(F_{1,27}=5.40, P=0.03\right)$ interactions. Therefore, the magnitude of the PAS effects as a function of aging and gender does not seem to be influenced by the between-group differences in the N20 latencies.

As suggested by the data presented in Fig. 1, there was a differential PAS effect on the N20 and P25 components. Aging and PAS affected the amplitude of the P25 component but are not associated with significant changes in the N20 amplitude (Table 1). In fact, the N20 component only showed a significant Age group $\times$ Condition interaction, pointing to an increased amplitude only in the elderly $(P=0.01)$ without significant changes in young subjects $(P=0.49)$. On the other hand, the P25 component was significantly affected by aging, with a higher amplitude in elderly $(3.06 \pm 0.51 \mu \mathrm{V})$ than young $(1.65 \pm 0.33 \mu \mathrm{V})$ subjects and was also affected by PAS, with an increased amplitude post-PAS (pre-PAS $=2.24 \pm 0.32 \mu \mathrm{V}$; post-PAS $=$ $2.47 \pm 0.33 \mu \mathrm{V}$ ). Gender differences did not affect the N20 and P25 components.

Finally, the PAS-induced changes were limited to the N20-P25 complex, since the ANOVAs of the amplitude of the P14, N30, and P40 components did not show any significant effects or interactions. Only aging affected the amplitude of the $\mathrm{P} 14$ (young $=0.93 \pm 0.12 \mu \mathrm{V}$, elderly $=1.27 \pm$ $0.11 \mu \mathrm{V} ; F_{1,30}=4.09, P=0.05$ ) and $\mathrm{P} 40$ components (young $=$ $2.23 \pm 0.34 \mu \mathrm{V}$, elderly $=5.34 \pm 0.50 \mu \mathrm{V} ; F_{1,30}=30.88, P<$ $0.001)$. The $P 40$ components also showed a main effect for Gender, with a higher amplitude in females $(4.43 \pm 0.62$ $\mu \mathrm{V})$ than males $(3.14 \pm 0.50 \mu \mathrm{V})$.

\section{DISCUSSION}

The present results confirm that low-frequency MN stimulation paired with TMS over the contralateral cortex enhances S1 excitability, as indicated by SEP analysis. The main finding of our study is that the S1 LTP-like plasticity after PAS intervention changes as a function of age, with a significant increase of the N20-P25 complex in elderly $(9.4 \%)$ compared to young subjects $(5.4 \%)$. Notably, the increase found by Wolters et al. (2005) in young subjects was very similar (6.2\%). Therefore, our results confirm previous studies (Wolters et al., 2005; Litvak et al., 2007) supporting the efficacy of this protocol in healthy young subjects to induce a significant, although of small extent, LTP-like plasticity in S1 cortex, and the need to follow the original experimental protocol, because even slight differences between the experimental protocols accounted for the lack of overt change in somatosensory cortical excitability after PAS, as highlighted in a previous study (Bliem et al., 2008).

Moreover, our PAS intervention induces an amplitude increase of N20 in elderly but not in young subjects and an increase in the amplitude of P25 in both age groups. At a statistical level, this increase persists despite the significant difference between age groups on N20 latency, an effect which is likely associated with a slowing of conduction afferent pathways in the elderly.

PAS does not induce modulation of P14, N30, and P40 components, although we confirm that these components were affected by aging (Kakigi and Shibasaki, 1991). This lack of significant changes after PAS intervention on the 
subcortically generated P14 (Desmedt and Cheron, 1980b; Lee and Seyal, 1998) supports an interpretation that excludes a contribution of subcortical mechanisms to somatosensory excitability, supporting the idea that the main site of action for PAS-induced synaptic plasticity is the cortical level (Stefan et al., 2002; Wolters et al., 2003) and that cortical excitability changes are due to modulation of excitatory neuronal activity in upper cortical layers of S1 (Wolters et al., 2005). However, further studies will be necessary to directly investigate a possible contribution of spinal mechanisms in excitability of the somatosensory system, as was recently shown in the motor system (Meunier et al., 2007).

Finally, this specificity also allows us to exclude that the increased PAS-induced plasticity effect could simply reflect between-group differences in baseline excitability (Rosenkranz et al., 2007), since differences in baseline excitability should likely affect all SEP components after PAS.

\section{Cortical plasticity and compensatory changes with aging on somatosensory cortex}

SEP components that are more affected by PAS protocol are also those more affected by age-dependent changes. It is now widely accepted that both early SEP components, $\mathrm{N} 20$ and P25, are generated in the posterior bank of the central sulcus and primarily reflect activity of S1 (Broughton et al., 1981; Hume and Cant, 1981; Allison et al., 1989, 1991; Urbano et al., 1997). Intracranial recordings in humans (Allison, 1982) indicate that $\mathrm{N} 20$ and P25 reflect the initial excitation of neurons in cortical areas $3 b$ and 1 , respectively, areas that also show a significant effect of aging (Allison et al., 1984). Therefore, we confirm an increased amplitude of the N20-P25 complex and P25 component in elderly compared to young subjects, independent of PAS intervention, since a general and gradual increase of SEP amplitude as well as of somatosensory evoked field has been repeatedly found in normal elderly subjects (Luders, 1970; Kazis et al., 1983; Kakigi and Shibasaki, 1991; Huttunen et al., 1999; Nakano and Hashimoto, 1999; Stephen et al., 2006). An ultimate explanation of this phenomenon is still lacking, but the foremost hypothesis is that this greater activation may be due (a) to the development of dendritic arborizations in the cortical neurons, which compensate for the degeneration of the synaptic spines (Wisniewski and Terry, 1976; Desmedt and Cheron, 1980a; Kazis et al., 1983) and (b) to a cortical decrease in the inhibitory neurotransmitters (GABA), resulting in a prevalence of excitatory intracortical mechanisms (Paty et al., 1980; Kazis et al., 1983). A decreased inhibition in the elderly has been suggested by animal studies (Drechsler, 1978; McDowd and Oseas-Kreger, 1991) showing an age-related decrease in the number of inhibitory synapses rather than a decrease in neuronal number in the aged S1 (Brunso-Bechtold et al., 2000). There is also evidence that normal aging in humans reduces cortical reciprocal inhibition (Hortobagyi et al., 2006) and is associated with a relative decrease in the excitability of intracortical inhibitory circuits (Peinemann et al., 2001).
As a matter of fact, different studies from various view angles (biochemical, neurophysiological, and clinical) indicate that aging impairs GABAergic inhibition in afferent relay nuclei and in the hippocampal cortex (Post-Munson et al., 1994; Caspary et al., 1995) and enhances glutamatergic excitation in the neocortex (Wenk et al., 1989; Saransaari and Oja, 1995). Therefore, aging could be associated with a change in the balance between excitation and inhibition, trending towards an increase in cortical excitability (Huttunen et al., 1999).

The amplitude increase of cortical evoked potentials in aged individuals, together with a decrease of conduction velocity in the peripheral somatosensory pathways and normal central conduction velocity, supports the view that aging does not simply cause a progressive degeneration of the nervous system. On the contrary, it can produce compensatory reactions deviating from the linear retrogression, possibly to actively resist the coming degeneration (Kazis et al., 1983).

Current results suggest that a larger SEP increase induced by PAS in elderly than in young subjects represents an additional and plastic increase in the strength of excitatory transmission. On this basis, it could be speculated that PAS intervention determinates a reduction of intracortical, presumably GABAergic, inhibition or an enhancement of intracortical, presumably glutamatergic, excitation or a mixture of both (Segovia et al., 2001; Ragert et al., 2004). Studies in human and non-human cortex confirmed that the induction of LTP effects should require a reduction in local cortical inhibition by modulation of GABA receptor activity (Hess and Donoghue, 1994) or an increase in NMDA receptor activation (Ziemann et al., 1998).

Additionally, another probable cause of the PAS-induced wider increase in both N20 and P25 in elderly could be a progressive decrease of the specific cortical inhibitory processes during aging (Drechsler, 1978). It is widely accepted that the N20 component represents the initial postsynaptic activity in the S1 cortex (Allison et al., 1989), while the P25 component is assumed to represent the arrival of the sensory volley in S1 (Rossini et al., 1987; Tsuji and Rothwell, 2002). The serial activation of areas $3 \mathrm{~b}$ and 1 (Jones et al., 1978; Kaas and Garraghty, 1991; Urbano et al., 1997) on a background of enhanced cortical excitability in conjunction with cortical inhibitory decrease could explain the greater LTP-like increase initially seen in N20 and later in P25 in elderly compared to young subjects. We hypothesize that the differential PAS-induced cortical excitability changes between young and elderly subjects could be a result of differential excitatory modulation and inhibitory involvement of neuronal activity in $\mathrm{S} 1$, which results in more confined and specific plasticity effects in young and more generalized and wider plasticity in the elderly.

One possible scenario is that the more generalized and wider plasticity in the elderly represents a compensatory mechanism, in terms of functional reserve of somatosensory cortex, activated by the aging brain to counterbalance cortical degeneration associated with aging (Craik, 2006; Heuninckx et al., 2008). According to the compensation 
hypothesis that supports the age-related increases in brain activation, as overactivation or greater brain activity, to compensate for various neurodegenerative deficits (Madden et al., 1999; Cabeza et al., 2002; Reuter-Lorenz and Lustig, 2005; Rossi et al., 2004), our results suggest that greater S1 LTP-like plasticity after PAS in elderly subjects is counteracted to age-related structural and neurochemical decline through greater plastic modulation of neurocortical pathways and reorganization of functional networks.

\section{Gender effects on cortical plasticity}

The results of our study highlight that plastic changes on S1 appear to be not only age-dependent but also genderdependent, confirming the growing evidence that neuroplastic changes are influenced by gender-specific pressure (Cahill, 2006) and presenting for the first time direct evidence of gender differences in human somatosensory cortical plasticity.

In particular, we point out that gender modulates the PAS-induced increase of the N20-P25 amplitude, with a significant and larger increase in the female elderly group. This gender difference seems further accentuated with aging, modulating neuroplasticity mechanisms that affect both neural excitability and cortical plasticity (Teskey et al., 1999; Inghilleri et al., 2004). Reasonably, the small, but not significant, PAS-induced effect on P25 amplitude found in normal adult subjects, serving as a control group in a recent study (Tamura et al., 2009), should also be explained by these gender differences. In fact, eight out of 10 subjects of that study were males.

In animal studies, sex differences in synaptic plasticity and cognitive functions have been widely demonstrated (Juraska, 1998; Jonasson, 2005) and sex hormones were suggested as modulating these effects (Woolley, 2000; McEwen, 2002). In fact, ovarian steroids influence neural excitability (Fink, 1994; Kawata, 1995), with excitability increasing due to estrogen and decreasing due to progesterone (Beyenburg et al., 2001).

Neuronal function and excitability can be affected by steroid hormones directly by modulating the activity of neurotransmitter receptors (GABA and NMDA receptors) or indirectly by modulating ion channels (Kawata, 1995; Rupprecht and Holsboer, 1999). More directly, the presence of estrogens in women could play an important role in synaptic potentiation and cortical excitability induced by PAS on S1 by enhancing glutamate action on NMDA receptors and by acting on voltage-gated sodium channels (Malenka and Nicoll, 1999; Inghilleri et al., 2004). This suggests that sex hormones, which already contribute to plastic changes in young females, could interact with the wider cortical excitability, which contributes to the greatest plasticity of the elderly compared to young. Moreover, we ascribe the plasticity effects on S1 to hormonal factors and not to the gender-specificities of brain structure, considering that gender itself does not affect the amplitude of SEP but only modulates the PAS-induced increase in SEP.

In accordance with these animal and human studies, our results also point to an effect of sexual dimorphism on human cortical somatosensory neuroplasticity, probably mediated by differences in sex hormones.

\section{Aging-related plastic changes in somatosensory vs. motor cortex}

Plastic changes induced by the PAS protocol seem to differentially affect the S1 and M1 parts of cortex with different age- and gender-dependent effects. Our study highlights the capability of S1 to retain plasticity during physiological aging. On the other hand, studies on M1 showed a decline in the capacity of this area to undergo plastic changes induced by PAS in elderly subjects (Muller-Dahlhaus et al., 2008). Moreover, it has been demonstrated (Tecchio et al., 2008) that PAS-induced motor cortex plasticity is also gender-dependent, with a decrease in plastic change in females after menopause. The former study correlated reduced M1 plasticity to several biochemical mechanisms and cortical-spinal functions affected by aging; while the latter hypothesized an imbalance of intracortical excitatory network activity that differentially affects plasticity in elderly females.

The results of these studies on PAS-induced synaptic plasticity on $\mathrm{M} 1$, though partially contrasting, both indicate that $\mathrm{S} 1$ and $\mathrm{M} 1$ have different plastic capability (CastroAlamancos et al., 1995). Although these different cortical areas have similar types of neurons and basic pattern of neural circuitry, they are different in their peripheral and cortical pathways, in their inputs and outputs, and in their functional and molecular properties (Bower and Haberly, 1986; Huntley et al., 1994; Castro-Alamancos and Connors, 1996; Barth, 2002). Previous studies have reported that S1 and M1 can reorganize in response to central or peripheral manipulations by expressing a variety of types of synaptic plasticity. Nevertheless, these cortices are not identical in their plastic change capabilities and in their ability to generate LTP (Donoghue et al., 1990; Recanzone et al., 1992; Diamond et al., 1993; Castro-Alamancos et al., 1995; Barth, 2002). Moreover, changes in the structural complexity and susceptibility to plasticity in both these areas appear differentially affected by aging (Adams, 1987a,b; Dickstein et al., 2007). In particular, studies on age-related changes in synaptic architecture highlight that aging is associated with a decrease in synapse number and an increase in the length of the postsynaptic contact zone in the M1 but not in S1 (Adams, 1987b).

It is now known that aging represents a physiological process that occurs asynchronously in different areas of the brain and that the rate of this process is related to the neuronal-synaptic-molecular substrates in each area (Mora et al., 2007). The differential age-related changes produced in several regions of the brain in the anatomy of neurons (Burke and Barnes, 2006), volume tissue density (Hedden and Gabrieli, 2004), and dynamics of several neurotransmitters (Segovia et al., 1999, 2001; Del Arco et al., 2003) support the hypothesis of the differential ability to make plastic changes in different cortical areas. Thus, the different structural and functional features of $S 1$ and $M 1$, and their age- and gender-related modification (Pitcher et al., 2003; Sale and Semmler, 2005; Stephen et al., 2006) 
can explain the differential ability of these cortices to make AQ: 4 plastic changes and can explain their involvement in the balance of the intracortical network and spinal activity.

Finally, the different effects of aging on S1 and M1 could be due to a different involvement in how PAS protocol recruits these two areas. Indeed, when PAS is applied to $\mathrm{S} 1$, it acts on more localized cortical mechanisms. On the contrary, PAS applied to M1 acts on more complex networks in the cortico-cortical pathway between S1 and M1 (Porter, 1997), which could be more affected by physiological and neurochemical mechanisms correlated with aging.

\section{Limitations of the study}

The main limitation of this study is the absence of a control condition for PAS intervention that allows us to attribute the increase of SEPs to the pairing of MN stimulation with TMS and to exclude the effects of elapsed time or prolonged MN stimulation. However, this possibility is unlikely considering that previous studies have already demonstrated the efficacy of the PAS protocol, at specific time intervals between peripheral and cortical stimulation, to induce somatosensory cortical changes (Wolters et al., 2005; Litvak et al., 2007).

Furthermore, it should be mentioned that, even though sensory perceptual and motor thresholds do not covariate for PAS-induced N20-P25 amplitude changes, this does not exclude a possible contribution of different thresholds across groups and the ultimate possibility that somatosensory and motor thresholds are not equally affected by aging.

Finally, relatively to gender-dependent factors, data regarding hormonal levels, menstrual cycle etc. were not collected at the time of experiment, since we were primarily focused on age factor and this should be considered a limitation of the study.

\section{CONCLUSION}

Our results confirm the efficacy of the PAS protocol in inducing plastic changes in S1 cortex and highlight that these effects are age- and gender-dependent. Specifically, our results are in agreement with the hypothesis that the greater plasticity seen in specific neuronal circuits in the elderly could represent a compensatory mechanism of functional reorganization for the aging brain that makes up for the physiologic and neurodegenerative decline correlated with aging. Moreover, the PAS protocol could represent a valuable tool to improve cortical functionality for sensorial ability that deteriorates during aging due to its ability to induce plastic changes on S1 in the elderly.

Acknowledgments-We thank Marinella Rosato for her help in data collection and Lottomatica, Azienda Autonoma Monopoli di Stato for financial support.

\section{REFERENCES}

AQ: 5 Adams I (1987a) Plasticity of the synaptic contact zone following loss of synapses in the cerebral cortex of aging humans. Brain Res 424:343-351.
Adams I (1987b) Comparison of synaptic changes in the precentral and postcentral cerebral cortex of aging humans: a quantitative ultrastructural study. Neurobiol Aging 8:203-212.

Allison T (1982) Scalp and cortical recordings of initial somatosensory cortex activity to median nerve stimulation in man. Ann N Y Acad Sci 388:671-678.

Allison T, Hume AL, Wood CC, Goff WR (1984) Developmental and aging changes in somatosensory, auditory and visual evoked potentials. Electroencephalogr Clin Neurophysiol 58:14-24.

Allison T, McCarthy G, Wood CC, Darcey TM, Spencer DD, Williamson PD (1989) Human cortical potentials evoked by stimulation of the median nerve. I. Cytoarchitectonic areas generating shortlatency activity. J Neurophysiol 62:694-710.

Allison T, McCarthy G, Wood CC, Jones SJ (1991) Potentials evoked in human and monkey cerebral cortex by stimulation of the median nerve. A review of scalp and intracranial recordings. Brain 114(Pt 6):2465-2503.

Barth AL (2002) Differential plasticity in neocortical networks. Physiol Behav 77:545-550.

Beyenburg S, Stoffel-Wagner B, Bauer J, Watzka M, Blumcke I, Bidlingmaier F, Elger CE (2001) Neuroactive steroids and seizure susceptibility. Epilepsy Res 44:141-153.

Bi GQ, Poo MM (1998) Synaptic modifications in cultured hippocampal neurons: dependence on spike timing, synaptic strength, and postsynaptic cell type. J Neurosci 18:10464-10472.

Bliem B, Muller-Dahlhaus JF, Dinse HR, Ziemann U (2008) Homeostatic metaplasticity in the human somatosensory cortex. J Cogn Neurosci 20:1517-1528.

Bower JM, Haberly LB (1986) Facilitating and nonfacilitating synapses on pyramidal cells: a correlation between physiology and morphology. Proc Natl Acad Sci U S A 83:1115-1119.

Broughton R, Rasmussen T, Branch C (1981) Scalp and direct cortical recordings of somatosensory evoked potentials in man. Can J Psychol 35:136-158.

Brunso-Bechtold JK, Linville MC, Sonntag WE (2000) Age-related synaptic changes in sensorimotor cortex of the Brown Norway $X$ Fischer 344 rat. Brain Res 872:125-133.

Burke SN, Barnes CA (2006) Neural plasticity in the ageing brain. Nat Rev Neurosci 7:30-40.

Cabeza R, Anderson ND, Locantore JK, McIntosh AR (2002) Aging gracefully: compensatory brain activity in high-performing older adults. Neuroimage 17:1394-1402.

Cahill L (2006) Why sex matters for neuroscience. Nat Rev Neurosci 7:477-484.

Caspary DM, Milbrandt JC, Helfert RH (1995) Central auditory aging: GABA changes in the inferior colliculus. Exp Gerontol 30:349-360.

Castro-Alamancos MA, Donoghue JP, Connors BW (1995) Different forms of synaptic plasticity in somatosensory and motor areas of the neocortex. J Neurosci 15:5324-5333.

Castro-Alamancos MA, Connors BW (1996) Short-term synaptic enhancement and long-term potentiation in neocortex. Proc Natl Acad Sci U S A 93:1335-1339.

Classen J, Wolters A, Stefan K, Wycislo M, Sandbrink F, Schmidt A, Kunesch E (2004) Paired associative stimulation. Suppl Clin Neurophysiol 57:563-569.

Craik FI (2006) Brain-behavior relations across the lifespan: a commentary. Neurosci Biobehav Rev 30:885-892.

Del Arco A, Segovia G, Fuxe K, Mora F (2003) Changes in dialysate concentrations of glutamate and GABA in the brain: an index of volume transmission mediated actions? J Neurochem 85:23-33.

Desmedt JE, Cheron G (1980a) Somatosensory evoked potentials to finger stimulation in healthy octogenarians and in young adults: wave forms, scalp topography and transit times of parietal and frontal components. Electroencephalogr Clin Neurophysiol 50:404425.

Desmedt JE, Cheron G (1980b) Central somatosensory conduction in man: neural generators and interpeak latencies of the far-field 
components recorded from neck and right or left scalp and earlobes. Electroencephalogr Clin Neurophysiol 50:382-403.

Diamond ME, Armstrong-James M, Ebner FF (1993) Experiencedependent plasticity in adult rat barrel cortex. Proc Natl Acad Sci U S A 90:2082-2086.

Dickstein DL, Kabaso D, Rocher AB, Luebke JI, Wearne SL, Hof PR (2007) Changes in the structural complexity of the aged brain. Aging Cell 6:275-284.

Donoghue JP, Suner S, Sanes JN (1990) Dynamic organization of primary motor cortex output to target muscles in adult rats. II. Rapid reorganization following motor nerve lesions. Exp Brain Res 79: 492-503.

Drechsler F (1978) Quantitative analysis of neurophysiological processes of the aging CNS. J Neurol 218:197-213.

Feldman DE (2000) Timing-based LTP and LTD at vertical inputs to layer II/III pyramidal cells in rat barrel cortex. Neuron 27:45-56.

Fink G (1994) Molecular principles from neuroendocrine models: steroid control of central neurotransmission. Prog Brain Res 100: 139-147.

Fratello F, Veniero D, Curcio G, Ferrara M, Marzano C, Moroni F, Pellicciari MC, Bertini M, Rossini PM, De Gennaro L (2006) Modulation of corticospinal excitability by paired associative stimulation: reproducibility of effects and intraindividual reliability. Clin Neurophysiol 117:2667-2674.

Hedden T, Gabrieli JD (2004) Insights into the ageing mind: a view from cognitive neuroscience. Nat Rev Neurosci 5:87-96.

Hess G, Donoghue JP (1994) Long-term potentiation of horizontal connections provides a mechanism to reorganize cortical motor maps. J Neurophysiol 71:2543-2547.

Heuninckx S, Wenderoth N, Swinnen SP (2008) Systems neuroplasticity in the aging brain: recruiting additional neural resources for successful motor performance in elderly persons. J Neurosci 28 : 91-99.

Hortobagyi T, del Olmo MF, Rothwell JC (2006) Age reduces cortical reciprocal inhibition in humans. Exp Brain Res 171:322-329.

Hume AL, Cant BR (1981) Central somatosensory conduction after head injury. Ann Neurol 10:411-419.

Huntley GW, Vickers JC, Morrison JH (1994) Cellular and synaptic localization of NMDA and non-NMDA receptor subunits in neocortex: organizational features related to cortical circuitry, function and disease. Trends Neurosci 17:536-543.

Huttunen J, Wikstrom H, Salonen O, IImoniemi RJ (1999) Human somatosensory cortical activation strengths: comparison between males and females and age-related changes. Brain Res 818: 196-203.

Inghilleri M, Conte A, Curra A, Frasca V, Lorenzano C, Berardelli A (2004) Ovarian hormones and cortical excitability. An rTMS study in humans. Clin Neurophysiol 115:1063-1068.

Jonasson Z (2005) Meta-analysis of sex differences in rodent models of learning and memory: a review of behavioral and biological data. Neurosci Biobehav Rev 28:811-825.

Jones EG, Coulter JD, Hendry SH (1978) Intracortical connectivity of architectonic fields in the somatic sensory, motor and parietal cortex of monkeys. J Comp Neurol 181:291-347.

Juraska JM (1998) Neural plasticity and the development of sex differences. Annu Rev Sex Res 9:20-38.

Kaas JH, Garraghty PE (1991) Hierarchical, parallel, and serial arrangements of sensory cortical areas: connection patterns and functional aspects. Curr Opin Neurobiol 1:248-251.

Kakigi R, Shibasaki H (1991) Effects of age, gender, and stimulus side on scalp topography of somatosensory evoked potentials following median nerve stimulation. J Clin Neurophysiol 8:320-330.

Kawata M (1995) Roles of steroid hormones and their receptors in structural organization in the nervous system. Neurosci Res 24:1-46.

Kazis A, Vlaikidis N, Pappa P, Papanastasiou J, Vlahveis G, Routsonis K (1983) Somatosensory and visual evoked potentials in human aging. Electromyogr Clin Neurophysiol 23:49-59.
Lee EK, Seyal M (1998) Generators of short latency human somatosensory-evoked potentials recorded over the spine and scalp. $\mathrm{J}$ Clin Neurophysiol 15:227-234.

Litvak V, Zeller D, Oostenveld R, Maris E, Cohen A, Schramm A, Gentner R, Zaaroor M, Pratt H, Classen J (2007) LTP-like changes induced by paired associative stimulation of the primary somatosensory cortex in humans: source analysis and associated changes in behaviour. Eur J Neurosci 25:2862-2874.

Luders H (1970) The effect of aging on the wave form of the somatosensory cortical evoked potential. Electroencephalogr Clin Neurophysiol 29:450-460.

Madden DJ, Turkington TG, Provenzale JM, Denny LL, Hawk TC, Gottlob LR, Coleman RE (1999) Adult age differences in the functional neuroanatomy of verbal recognition memory. Hum Brain Mapp 7:115-135.

Malenka RC, Nicoll RA (1993) NMDA-receptor-dependent synaptic plasticity: multiple forms and mechanisms. Trends Neurosci 16: 521-527.

Malenka RC, Nicoll RA (1999) Long-term potentiation: a decade of progress? Science 285:1870-1874.

Mariorenzi R, Zarola F, Caramia MD, Paradiso C, Rossini PM (1991) Non-invasive evaluation of central motor tract excitability changes following peripheral nerve stimulation in healthy humans. Electroencephalogr Clin Neurophysiol 81:90-101.

Markram H, Lubke J, Frotscher M, Sakmann B (1997) Regulation of synaptic efficacy by coincidence of postsynaptic APs and EPSPs. Science 275:213-215.

McDowd JM, Oseas-Kreger DM (1991) Aging, inhibitory processes, and negative priming. J Gerontol 46:P340-P345.

McEwen B (2002) Estrogen actions throughout the brain. Recent Prog Horm Res 57:357-384.

Mercado E 3rd (2008) Neural and cognitive plasticity: from maps to minds. Psychol Bull 134:109-137.

Meunier S, Russmann H, Simonetta-Moreau M, Hallett M (2007) Changes in spinal excitability after PAS. J Neurophysiol 97:31313135.

Mora F, Segovia G, del Arco A (2007) Aging, plasticity and environmental enrichment: structural changes and neurotransmitter dynamics in several areas of the brain. Brain Res Rev 55:78-88.

Muller-Dahlhaus JF, Orekhov Y, Liu Y, Ziemann U (2008) Interindividual variability and age-dependency of motor cortical plasticity induced by paired associative stimulation. Exp Brain Res 187: 467-475.

Murakami T, Sakuma K, Nomura T, Uemura Y, Hashimoto I, Nakashima K (2008) Changes in somatosensory-evoked potentials and high-frequency oscillations after paired-associative stimulation. Exp Brain Res 184:339-347.

Nakano S, Hashimoto I (1999) The later part of high-frequency oscillations in human somatosensory evoked potentials is enhanced in aged subjects. Neurosci Lett 276:83-86.

Nuwer MR, Aminoff M, Desmedt J, Eisen AA, Goodin D, Matsuoka S, Mauguiere F, Shibasaki H, Sutherling W, Vibert JF (1994) IFCN recommended standards for short latency somatosensory evoked potentials. Report of an IFCN committee. International Federation of Clinical Neurophysiology. Electroencephalogr Clin Neurophysiol 91:6-11.

Oliviero A, Profice P, Tonali PA, Pilato F, Saturno E, Dileone M, Ranieri F, Di Lazzaro V (2006) Effects of aging on motor cortex excitability. Neurosci Res 55:74-77.

Paty J, Gioux M, Deliac P, Gros C, Claverie B, Lagenie M, Emeriau JP, Galley P (1980) [Visual evoked potentials in elderly subjects (author's transl)]. Rev Electroencephalogr Neurophysiol Clin 10:161170.

Peinemann A, Lehner C, Conrad B, Siebner HR (2001) Age-related decrease in paired-pulse intracortical inhibition in the human primary motor cortex. Neurosci Lett 313:33-36. 
Pitcher JB, Ogston KM, Miles TS (2003) Age and sex differences in human motor cortex input-output characteristics. J Physiol 546: $605-613$.

Porter LL (1997) Morphological characterization of a cortico-cortical relay in the cat sensorimotor cortex. Cereb Cortex 7:100-109.

Post-Munson DJ, Lum-Ragan JT, Mahle CD, Gribkoff VK (1994) Reduced bicuculline response and GABAA agonist binding in aged rat hippocampus. Neurobiol Aging 15:629-633

Ragert P, Becker M, Tegenthoff M, Pleger B, Dinse HR (2004) Sustained increase of somatosensory cortex excitability by $5 \mathrm{~Hz}$ repetitive transcranial magnetic stimulation studied by paired median nerve stimulation in humans. Neurosci Lett 356:91-94.

Recanzone GH, Merzenich MM, Dinse HR (1992) Expansion of the cortical representation of a specific skin field in primary somatosensory cortex by intracortical microstimulation. Cereb Cortex 2: 181-196.

Reuter-Lorenz PA, Lustig C (2005) Brain aging: reorganizing discoveries about the aging mind. Curr Opin Neurobiol 15:245-251.

Rioult-Pedotti MS, Friedman D, Hess G, Donoghue JP (1998) Strengthening of horizontal cortical connections following skill learning. Nat Neurosci 1:230-234.

Rosenkranz K, Kacar A, Rothwell JC (2007) Differential modulation of motor cortical plasticity and excitability in early and late phases of human motor learning. J Neurosci 27:12058-12066.

Rossi S, Miniussi C, Pasqualetti P, Babiloni C, Rossini PM, Cappa SF (2004) Age-related functional changes of prefrontal cortex in longterm memory: a repetitive transcranial magnetic stimulation study. J Neurosci 24:7939-7944.

Rossini PM, Gigli GL, Marciani MG, Zarola F, Caramia M (1987) Non-invasive evaluation of input-output characteristics of sensorimotor cerebral areas in healthy humans. Electroencephalogr Clin Neurophysiol 68:88-100.

Rossini PM, Martino G, Narici L, Pasquarelli A, Peresson M, Pizzella V, Tecchio F, Torrioli G, Romani GL (1994a) Short-term brain "plasticity" in humans: transient finger representation changes in sensory cortex somatotopy following ischemic anesthesia. Brain Res 642:169-177.

Rossini PM, Barker AT, Berardelli A, Caramia MD, Caruso G, Cracco $R Q$, Dimitrijevic MR, Hallett M, Katayama Y, Lucking $\mathrm{CH}$, et al. (1994b) Non-invasive electrical and magnetic stimulation of the brain, spinal cord and roots: basic principles and procedures for routine clinical application. Report of an IFCN committee. Electroencephalogr Clin Neurophysiol 91:79-92.

Rossini PM, Rossi S, Babiloni C, Polich J (2007) Clinical neurophysiology of aging brain: from normal aging to neurodegeneration. Prog Neurobiol 83:375-400.

Rupprecht R, Holsboer F (1999) Neuroactive steroids: mechanisms of action and neuropsychopharmacological perspectives. Trends Neurosci 22:410-416.

Sale MV, Semmler JG (2005) Age-related differences in corticospinal control during functional isometric contractions in left and right hands. J Appl Physiol 99:1483-1493.

Sale MV, Ridding MC, Nordstrom MA (2007) Factors influencing the magnitude and reproducibility of corticomotor excitability changes induced by paired associative stimulation. Exp Brain Res 181: 615-626.

Sanes JN, Donoghue JP (2000) Plasticity and primary motor cortex. Annu Rev Neurosci 23:393-415.

Sankoh AJ, Huque MF, Dubey SD (1997) Some comments on frequently used multiple endpoint adjustments methods in clinical trials. Stat Med 16:2529-2542.

Saransaari P, Oja SS (1995) Age-related changes in the uptake and release of glutamate and aspartate in the mouse brain. Mech Ageing Dev 81:61-71.
Segovia G, Del Arco A, Mora F (1999) Effects of aging on the interaction between glutamate, dopamine, and GABA in striatum and nucleus accumbens of the awake rat. J Neurochem 73:20632072.

Segovia G, Porras A, Del Arco A, Mora F (2001) Glutamatergic neurotransmission in aging: a critical perspective. Mech Ageing Dev 122:1-29.

Stavrinou ML, Della Penna S, Pizzella V, Torquati K, Cianflone F, Franciotti R, Bezerianos A, Romani GL, Rossini PM (2007) Temporal dynamics of plastic changes in human primary somatosensory cortex after finger webbing. Cereb Cortex 17:2134-2142.

Stefan K, Kunesch E, Benecke R, Cohen LG, Classen J (2002) Mechanisms of enhancement of human motor cortex excitability induced by interventional paired associative stimulation. J Physiol 543:699708.

Stefan K, Wycislo M, Classen J (2004) Modulation of associative human motor cortical plasticity by attention. J Neurophysiol 92: $66-72$.

Stephen JM, Ranken D, Best E, Adair J, Knoefel J, Kovacevic S, Padilla D, Hart B, Aine CJ (2006) Aging changes and gender differences in response to median nerve stimulation measured with MEG. Clin Neurophysiol 117:131-143.

Tamura Y, Ueki Y, Lin P, Vorbach S, Mima T, Kakigi R, Hallett M (2009) Disordered plasticity in the primary somatosensory cortex in focal hand dystonia. Brain 132(Pt 3):749-755.

Tecchio F, Zappasodi F, Pasqualetti P, Gennaro LD, Pellicciari MC, Ercolani M, Squitti R, Rossini PM (2008) Age dependence of primary motor cortex plasticity induced by paired associative stimulation. Clin Neurophysiol 119:675-682.

Teskey GC, Hutchinson JE, Kolb B (1999) Sex differences in cortical plasticity and behavior following anterior cortical kindling in rats. Cereb Cortex 9:675-682.

Tsuji T, Rothwell JC (2002) Long lasting effects of rTMS and associated peripheral sensory input on MEPs, SEPs and transcortical reflex excitability in humans. J Physiol 540:367-376.

Urbano A, Babiloni F, Babiloni C, Ambrosini A, Onorati P, Rossini PM (1997) Human short latency cortical responses to somatosensory stimulation. A high resolution EEG study. Neuroreport 8:32393243.

Wassermann EM (1998) Risk and safety of repetitive transcranial magnetic stimulation: report and suggested guidelines from the International Workshop on the Safety of Repetitive Transcranial Magnetic Stimulation, June 5-7, 1996. Electroencephalogr Clin Neurophysiol 108:1-16.

Wenk GL, Pierce DJ, Struble RG, Price DL, Cork LC (1989) Agerelated changes in multiple neurotransmitter systems in the monkey brain. Neurobiol Aging 10:11-19.

Wisniewski HM, Terry RD (1976) Neuropathology of aging brain. In: Neurobiology of aging (Terry RD, Gershon S, eds), pp 1-22. New York: Raven Press.

Wolters A, Sandbrink F, Schlottmann A, Kunesch E, Stefan K, Cohen LG, Benecke R, Classen J (2003) A temporally asymmetric Hebbian rule governing plasticity in the human motor cortex. J Neurophysiol 89:2339-2345.

Wolters A, Schmidt A, Schramm A, Zeller D, Naumann M, Kunesch E, Benecke R, Reiners K, Classen J (2005) Timing-dependent plasticity in human primary somatosensory cortex. J Physiol 565: 1039-1052

Woolley CS (2000) Effects of oestradiol on hippocampal circuitry. Novartis Found Symp 230:173-180; discussion: 181-177.

Ziemann U, Hallett M, Cohen LG (1998) Mechanisms of deafferentation-induced plasticity in human motor cortex. J Neurosci 18 : 7000-7007.

Ziemann U (2004) LTP-like plasticity in human motor cortex. Suppl Clin Neurophysiol 57:702-707. 